\title{
Tuberculosis y SIDA en Pediatría: A propósito de cinco casos
}

\author{
Julia Villarroel B., Eloisa Vizueta R., Ana M. Álvarez P., M. Isabel Galaz L., Anamaría Peña D., Patty Schuffeneger R. \\ y Ana Chávez P., en representación del Comité Nacional de SIDA Pediátrico
}

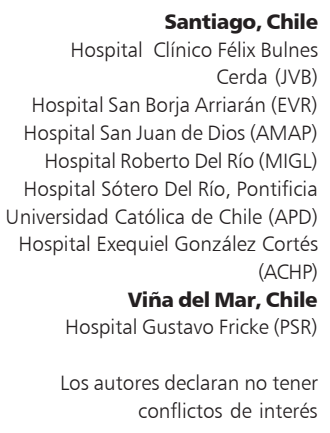

Recibido: 20 de diciembre de 2006 Aceptado: 4 de octubre de 2007

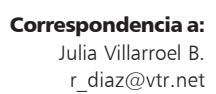

\section{Introducción}

$\mathrm{L}$ a tuberculosis (TBC) es una enfermedad endémica en la mayoría de los países en desarrollo, que se creía controlada; sin embargo, en las últimas décadas, ha reaparecido producto de varios factores, siendo la infección por VIH uno de los más importantes, sumado a la pobreza, la limitada atención en salud, el hacinamiento y las constantes migraciones ${ }^{1}$.

Los sujetos infectados por VIH tienen cinco a siete veces mayor posibilidad de adquirir TBC que la población general, en la que la TBC tiene una frecuencia de 3 a $5 \%$. Cuando coexisten ambas infecciones, la frecuencia de TBC alcanza a $17-37 \%$ en los cuatro meses siguientes a la exposición.

Estudios efectuados en la edad pediátrica han demostrado que los niños con TBC tienen mayor frecuencia de SIDA y en ellos, la mortalidad y las fallas de tratamiento son considerablemente mayores que en los niños seronegativos ${ }^{6}$.

El objetivo de este reporte es describir los casos controlados y manejados con TBC y SIDA que atiende el Comité Nacional de SIDA Pediátrico y su evolución en el tiempo.

Casuistica. En un total de 246 niños que adquirieran el VIH por transmisión vertical, que controló el Comité de SIDA pediátrico hasta marzo de 2006, se han producido seis casos de co-infección con TBC, cinco pertenecientes a la Región Metropolitana y uno a la Región de Valparaíso. Es importante considerar que no hay un protocolo para la pesquisa sistemática de TBC, sólo frente a la sospecha clínica, se inicia el estudio.
Sus edades fluctuaron entre 4 y 160 meses (promedio 40,8 meses), siendo la distribución igual para ambos sexos.

Al momento del diagnóstico de la infección por $\mathrm{VIH}$, tres niños se encontraban en etapa clínica A (dos A1 y uno A3), uno en etapa B3 y dos en etapa C (C1 y C3) (clasificación de CDC 1994). La edad al diagnosticarse la TBC varió entre cuatro y 161 meses (promedio 86,5 meses). En un caso (caso 6) el paciente debutó con TBC a los cuatro meses de vida y por ello se sospechó la infección por VIH, la que se confirmó ya en etapa C3 (Tabla 1).

Todos los niños habían recibido la vacuna BCG al nacer. Cinco habían tenido contacto en el hogar con un adulto enfermo de TBC. Cuando se diagnosticó la TBC, en cuatro de los seis niños había una progresión clínica e inmunológica, los dos casos restantes se mantenían en iguales condiciones (casos 2 y 6).

Sólo tres de los seis niños estaban con terapia antiretroviral, dos con biterapia (AZT, DDI) y uno triterapia (DDI, ABC, NVP). En dos de los niños con biterapia, ésta se suspendió al iniciar el tratamiento anti-tuberculoso (Tabla 2).

En todos los casos, la TBC se manifestó con compromiso pulmonar, agregándose efusión pleural en tres y en uno de ellos destacaba, además, la presencia de adenopatías mediastínicas. Las imágenes radiológicas fueron variadas: infiltrados parahiliares, adenopatías mediastínicas, imágenes de consolidación y efusión pleural (Tabla 3).

El diagnóstico se efectuó por cultivo de fluidos en cinco (lavado bronco-alveolar en tres niños, baciloscopia de contenido gástrico en uno, de líquido pleural en otro) y biopsia de adenopatías en un caso. En uno 
de los niños (caso 4) fue posible efectuar, además, RPC para Mycobacterium tuberculosis en contenido bronquial, la que resultó positiva (Tabla 3). El PPD se efectuó en cinco pacientes, siendo positivo al momento del diagnóstico sólo en cuatro niños (Tabla 2).

Con respecto a la terapia anti-tuberculosa, en tres casos se usó isoniazida, rifampicina y pirazinamida; en dos casos se agregó a la terapia convencional etambutol y en otro (caso 5), que recibía nevirapina como parte de su terapia antiretroviral, la que no se suspendió, se usó isoniazida, pirazinamida, etambutol y ciprofloxacina. El tratamiento anti-tuberculoso se mantuvo por 12 meses en todos los pacientes.

Se efectuó estudio de resistencia en dos de los seis casos, los que demostraron susceptibilidad a todas los fármacos anti-tuberculosos.

No se pesquisaron efectos adversos a la terapia específica, ni se observó el síndrome de reconstitución inmune.

Todos los casos evolucionaron en forma satisfactoria, con una sobrevida post TBC, al cierre del estudio, entre 7 y 132 meses (promedio: 55,3 meses). Uno de los pacientes (caso 2) está aún en tratamiento antituberculoso en su fase bisemanal (Tabla 4).

\section{Discusión}

La TBC es un problema de salud pública de tal magnitud y trascendencia que la OMS ha determinado que la meta de erradicar la TBC en el año 2000 se postergará varios años y lo ha declarado como una emergencia global. Se estima que un tercio de la población mundial (dos mil millones de personas) porta el bacilo de Koch en forma latente, se originan más de ocho millones de casos nuevos por año, de los cuales 800 mil son niños y que la TBC es responsable de más de tres millones de muertes por año ${ }^{1}$. En el continente americano también se aprecia un aumento de la incidencia de alrededor de $13 \%$, presentando los mayores problemas México ( $\uparrow 49 \%$ ), la región andina ( $\uparrow 30 \%$ ) y Brasil (个 23\%).

En Chile, la morbilidad y mortalidad ha ido en descenso a todas las edades (incidencia 18,4/100.000) ${ }^{2}$. La incidencia de la asociación TBC e infección por VIH es de $2,4 \%$, lo que coincide con las tasas nacionales vistas en niños chilenos, sin infección por VIH, con $\mathrm{TBC}^{2}$, a diferencia de elevadas tasas encontradas en otros países latinoamericanos y del Caribe, como Bolivia, Perú o Haití.

Recientemente, la infección por VIH se ha sumado a la larga lista de factores de riesgo para contraer la TBC; en la actualidad, constituye el factor primordial para contraer la TBC y se describe un gran riesgo de morta-

\begin{tabular}{|ccccc|}
\hline $\begin{array}{c}\text { Tabla 1. Edades y etapas clínico inmunológicas al diagnóstico de infección } \\
\text { por VIH } \mathbf{y} \text { tuberculosis } \\
\text { número }\end{array}$ & $\begin{array}{c}\text { Edad al } \\
\text { diagnóstico de } \\
\text { infección por } \\
\text { VIH (meses) }\end{array}$ & $\begin{array}{c}\text { Etapa al } \\
\text { diagnóstico de } \\
\text { infección por } \\
\text { VIH* }\end{array}$ & $\begin{array}{c}\text { Edad al } \\
\text { diagnóstico de } \\
\text { TBC (meses) }\end{array}$ & $\begin{array}{c}\text { Etapa al } \\
\text { (miagnóstico de } \\
\text { TBC* }\end{array}$ \\
\hline 1 & 30 & A1 & 108 & B3 \\
\hline 2 & 160 & B3 & 161 & B3 \\
\hline 3 & 22 & A3 & 72 & C3 \\
\hline 4 & 25 & A1 & 66 & B1 \\
\hline 5 & 4 & C1 & 108 & C3 \\
\hline 6 & 4 & C3 & 4 & \\
\hline *Según clasificación CDC 1994 & & & \\
\hline
\end{tabular}

\begin{tabular}{|c|c|c|c|c|c|}
\hline & & Contacto TBC & BCG & PPD & TARV \\
\hline Caso & 1 & Padres & Positivo & No se hizo & No \\
\hline Caso & 2 & Padres & Positivo & Positivo & No \\
\hline Caso & 3 & Padres & Positivo & Positivo & $A Z T+D D I$ \\
\hline Caso & 4 & Padres & Positivo & Positivo & $A Z T+D D I$ \\
\hline Caso & 5 & Padre & Positivo & Negativo & $\mathrm{DDI}+\mathrm{ABC}+\mathrm{NVP}$ \\
\hline Caso & 6 & No & Positivo & Positivo & No \\
\hline
\end{tabular}

Tabla 3. Órgano comprometido y exámenes diagnósticos

\begin{tabular}{|c|c|c|c|}
\hline & $\begin{array}{l}\text { Órgano } \\
\text { comprometido }\end{array}$ & Diagnóstico & $\begin{array}{l}\text { Radiografía de } \\
\text { tórax }\end{array}$ \\
\hline Caso 1 & Pleura y pulmón & LBA y cultivo & Consolidación \\
\hline Caso 2 & Pleura & Cultivo de líquido pleural & Efusión pleural \\
\hline Caso 3 & Pleura y adenopatías & Biopsia y cultivo & $\begin{array}{l}\text { Adenopatías } \\
\text { mediastínicas y efusión }\end{array}$ \\
\hline Caso 4 & Pulmón & LBA, cultivo y RPC & Consolidación \\
\hline Caso 5 & Pulmón & Baciloscopias y cultivo & $\begin{array}{l}\text { Infiltrados } \\
\text { paratraqueales }\end{array}$ \\
\hline Caso 6 & Pulmón & LBA, cultivo & Infiltrados perihiliares \\
\hline
\end{tabular}

\begin{tabular}{|lccccc|}
\hline \multicolumn{7}{|c|}{ Tabla 4. Terapia antituberculosa } \\
\hline Caso 1 & $\begin{array}{c}\text { Tratamiento } \\
\text { TBC }\end{array}$ & $\begin{array}{c}\text { Duración } \\
\text { (meses) }\end{array}$ & Resistencia & $\begin{array}{c}\text { Efectos } \\
\text { adversos }\end{array}$ & $\begin{array}{c}\text { Sobrevida } \\
\text { (meses) }\end{array}$ \\
Caso 2 & HIN, R, PZM, E & 12 & No estudiada & no & 36 \\
Caso 3 & HIN, R, PZM & 12 & No estudiada & no & 7 \\
Caso 4 & HIN, R, PZM & 12 & no & no & 75 \\
\hline Caso 5 & HIN, E, PZM, Cip & 12 & no & no & 132 \\
Caso 6 & HIN, R, PZM & 12 & no & no & 24 \\
\hline HIN: isoniazida, R: rifampicina, PZM: pirazinamida, E: etambutol, Cip: ciprofloxacina \\
\hline
\end{tabular}


lidad con la asociación de ambas infecciones. Durante el año 2002 hubo 600 mil niños muertos por SIDA en el mundo, de los cuales 200 mil estaban co- infectados con $\mathrm{TBC}^{3}$.

La mayor susceptibilidad de los pacientes infectados con VIH para contraer TBC se ha relacionado con la disminución en los linfocitos T1 y, por lo tanto, disminución también en la producción de interferón gama. Por otra parte, hay una profunda alteración funcional del sistema macrofágico y $M$. tuberculosis induce al macrófago a producir FNT, IL1 e IL6, sustancias pro inflamatorias que van a ocasionar una mayor replicación viral de VIH en las zonas infectadas por el bacilo tuberculoso ${ }^{4,5}$.

Estudios efectuados en niños demuestran que aquellos que cursan con TBC pueden tener, con mayor frecuencia, infección por $\mathrm{VIH}^{4}$ y fallas de tratamiento ${ }^{6,7}$.

Nuestros pacientes presentaron consolidaciones pulmonares y adenopatías mediastínicas, a pesar de tener tres de ellos un compromiso grave de la inmunidad (LT CD4 de 2, 4 y 11\%, respectivamente), lo que contrasta con lo descrito en la literatura, en que con niveles muy bajos de CD4, aparecen cuadros atípicos, con formas miliares y localizaciones extra-pulmonares $(36 \%)$ como son el compromiso del SNC (15\%) o TBC de piel y osteo-articular, dos formas que han resurgido en los últimos años, en asociación con la pandemia de SIDA $^{8,9}$

Estudios efectuados en niños demuestran que los síntomas más frecuentes son fiebre $(75 \%)$ mantenida por más de una semana, taquipnea, adenopatías periféricas, pérdida de peso superior a $10 \%$, mantenida por más de tres meses y la persistencia de signología pulmonar, a pesar de tratamientos convencionales bien llevados ${ }^{10,11}$

En nuestro país no ha habido casos de TBC congénita, una forma rara, pero que se presenta en niños nacidos de madres seropositivas para $\mathrm{VIH}$, con TBC activa. Esta forma puede resultar de la diseminación hematógena secundaria a micobacteriemia materna, rotura de un tubérculo placentario en la circulación fetal o por la deglución in útero de líquido amniótico infectado. La madre puede no tener síntomas de TBC y estar cursando una forma subclínica genital, resultando un RN infectado con síntomas inespecíficos, como inapetencia, falla en el crecimiento, hepatoesplenomegalia, fiebre, neumonía, meningitis y en ocasiones, puede debutar con un cuadro de distress respiratorio, apneas, ictericia y distensión abdominal ${ }^{12}$.

El diagnóstico de $\mathrm{TBC}$ en niños es siempre difícil, más aún, si co-existe con infección por VIH. Las baciloscopias, tienen, en general, baja sensibilidad (30-60\%) y especificidad $(50 \%)$, ya que a esta edad la infección es poco bacilífera y este examen requiere para visualizar el bacilo de Koch, entre 5.000 y 10.000 bacilos/ml de muestra; por lo tanto, una baciloscopia negativa no descarta el diagnóstico. En uno de nuestros pacientes, la baciloscopia en contenido gástrico resultó positiva; sin embargo, en los otros tres fue necesario efectuar lavado bronco-alveolar (con lo cual, se logra aumentar la positividad del examen a $80-90 \%$ ). La baciloscopia no permite diferenciar entre $M$. tuberculosis y otros bacilos atípicos, es fundamental efectuar siempre cultivo, ya sea con métodos tradicionales, en medio de Loewenstein-Jenssen, método que requiere de observación no menor a ocho semanas, o en medios líquidos radiométricos, que acortan el tiempo a alrededor de diez días. Cuando las baciloscopias son positivas, ambos tipos de cultivo tienen similar rendimiento, pero si las baciloscopias son negativas o en las formas extra-pulmonares, el cultivo con medio líquido tiene mejor rendimiento ${ }^{13-16}$

En sólo uno de nuestros pacientes se pudo efectuar RPC ya que, es costosa, no reemplaza a los métodos tradicionales y está aprobada por la FDA para uso sólo en muestras respiratorias o en pacientes que han recibido menos de siete días de tratamiento anti-tuberculoso. Su sensibilidad y especificidad es de $95 \%$ cuando las baciloscopias son positivas, pero si éstas son negativas, la sensibilidad disminuye a menos de $50 \%$; en consecuencia, sólo debe ser usada cuando la sospecha clínica es alta. Resultados de RPC en contenido gástrico han sido evaluados con una sensibilidad entre 45 y $83 \%$; puede dar falsos positivos con el complejo $M$. avium-intracelullare ${ }^{17}$.

La radiografía de tórax se aprecia alterada en $91 \%$ de los casos de TBC pulmonar ${ }^{17}$ con infiltrados pulmonares intersticiales difusos o localizados muy semejantes a los descritos en la neumocistosis, linfoadenopatías torácicas $\mathrm{y}$, en $25 \%$ de los niños, puede haber compromiso de múltiples lóbulos, dependiendo del estado inmunológico. La presencia de efusión pleural, observada en algunos de nuestros pacientes, se describe en $22 \%$ de los casos ${ }^{18}$.

El PPD se describe positivo sólo en 10 a $35 \%$ de los casos, por lo que no constituye una ayuda diagnóstica. Puede ser negativo debido a la anergia de estos pacientes y por lo tanto, no excluye el diagnóstico de $\mathrm{TBC}^{17}$.

En tuberculosis ganglionar y en el compromiso hepático, el estudio histológico es el elemento de mayor valor, ya que puede identificar bacilos por visualización directa y la presencia de granulomas en diferentes grados de constitución. La confirmación etiológica debe efectuarse por cultivo, ya que la identificación de granulomas es sugerente del diagnóstico pero no patognomónica de TBC. 
En cinco de nuestros pacientes se usó el esquema habitual de isoniazida, rifampicina, pirazinamida y etambutol. En uno solo se cambió rifampicina por ciprofloxacina por estar usando terapia con nevirapina. Es conocido que rifampicina induce actividad del citocromo P450, por lo cual, disminuye la concentración de inhibidores de proteasas y de inhibidores de transcriptasa reversa no nucleósidos; como consecuencia, disminuyen los niveles plasmáticos de efavirenz en 15 a $20 \%$ y de nevirapina en $30 \%$; con esta última se potencia, además, la hepatotoxicidad ${ }^{11,17,19,20}$. En pacientes infectados por VIH y TBC ocasionada por bacilos susceptibles, se recomienda tratarlos con tres fármacos, a lo menos dos meses y luego dos fármacos durante 9 a 12 meses. Si, por el contrario, la clínica y la respuesta bacteriológica no son buenas, el tratamiento debe prolongarse más de 12 meses, o mantenerse hasta cuatro meses después que los cultivos se negativizan. En general, en las formas pulmonares graves o en localizaciones extrapulmonares, se deben administrar cuatro anti-tuberculosos durante dos meses y luego tres fármacos por doce meses o más, incluso hasta dos años si los bacilos son resistentes ${ }^{20,21}$.

Nuestros pacientes recibieron profilaxis al estar en contacto con un adulto infectado. La profilaxis se describe efectiva para reducir la morbimortalidad por TBC en los pacientes con infección por VIH, pero el efecto protector desaparece rápidamente una vez que es suspendida. También disminuye la incidencia de TBC y la mortalidad en pacientes con infección por VIH con PPD $(+)$, pero no incide significativamente en los seropositivos con PPD $(-)^{21}$.

Efectos adversos de la terapia anti-tuberculosa. En ninguno de nuestros pacientes se pesquisaron efectos adversos conocidos, como elevación de transaminasas hepáticas o ictericia; este compromiso es más frecuente con isoniazida ( 10 a $20 \%$ ) y se manifiesta en 70 a 90 días de iniciado el tratamiento. En cambio, el compromiso hepático se ve en 1 a $4 \%$ de los pacientes tratados con rifampicina, y aparece tras ocho a 14 días de terapia. En ambos casos, se debería suspender el fármaco. Reacciones de tipo inmune, alergias, toxicidad auditiva, renal o polineuritis son descritas con menor frecuencia ${ }^{21}$ y no fueron observadas en esta serie clínica.
Síndrome de reconstitución inmune. Se caracteriza por la reaparición de la fiebre, adenopatías, infiltrados pulmonares y alteraciones neurológicas. Puede ocurrir en los primeros quince días de iniciado el tratamiento -en nuestros pacientes no se manifestó- describiéndose su aparición en $36 \%$ de los que reciben TARV en conjunto con el tratamiento anti-tuberculoso, versus $7 \%$ de los que reciben terapia anti-tuberculosa sola ${ }^{22-24}$. $\mathrm{Su}$ mayor incidencia se concentra en pacientes que inician TARV con CD4 muy bajos, menores de 50 células $/ \mathrm{mm}^{3}$.

Resistencia a fármacos anti-tuberculosos. No se presentó en los casos estudiados. La resistencia ha sido reportada por el CDC y la OMS, en forma creciente, a partir de 1990 y se observa tanto para isoniazida como para rifampicina; en el último tiempo se ha descrito, incluso, en medicamentos de segunda línea ${ }^{25,26}$. La resistencia a rifampicina es más frecuente en los pacientes infectados por VIH que en los no infectados y se produce por mutaciones en las cepas sensibles. En Chile, se estudia la resistencia in vitro desde 1971, tanto la primaria como la adquirida $\mathrm{y}$, afortunadamente, se mantiene en una frecuencia baja; estudios extranjeros muestran, por el contrario, una resistencia global que fluctúa entre 20 y $25 \%^{11,20,25}$.

En esta serie no se registró mortalidad; sin embargo, estudios efectuados en la edad pediátrica describen cifras altas de hasta $23 \%$ cuando co-existen ambas infecciones, versus $4 \%$ si los pacientes son seronegativos y sólo presentan TBC; la mayor mortalidad se produce en el grupo con LT CD4 inferior a $10 \%{ }^{1}$.

\section{Resumen}

La reemergencia de la tuberculosis (TBC) a nivel mundial se vincula a diferentes factores, como la infección por VIH y la inmunosupresión a la cual ésta conduce. Este comportamiento epidemiológico también se aprecia en la población infantil. Describimos la evolución clínica, inmunológica y tratamiento recibido por seis niños con infección VIH de transmisión vertical, controlados por el Comité Nacional de SIDA pediátrico, que presentaron tuberculosis. Se efectúa, además, una revisión de la literatura médica.

\section{Referencias}

1.- De Cock K, Binkin N, Zuber P, Tappero J, Castro K. Research issues involving HIVassociated tuberculosis in resource- poor countries. JAMA 1996; 276: 1502-7.

2.- Programa Nacional de Control de la
Tuberculosis 2005. Manual de organización y normas técnicas. Ministerio de Salud.

Gobierno de Chile.

3.- Donald P. Childhood tuberculosis: out of control? Cur Opin Pulmonary Med 2002; 8: 178-82.

4.- Kaufmann S. Protection against tuberculosis: cytokines, T cells, and macrophages. Ann Rheumat Dis 2002; 61 (Suppl 11): ii54-ii58.

5.- Jo E, Park J, Dockrell H. Dynamics of cytokine generation in patients with active pulmonary tuberculosis. Curr Opin Infect Dis 2003; 16: 205-10.

6.- Mukadi Y, Wiktor S, Coulibaly I, 
Coulibaly D, Mbengue A, Folquet A, et al. Impact of HIV infection on the development, clinical presentation, and outcome of tuberculosis among children in Abidjan, Cote d'Ivoire. AIDS 1997; 11: 1151-8.

7.- Espinal M, Reingold A, Pérez G, Camilo E, Soto S, Cruz E, et al. Human immunodeficiency virus infection in children with tuberculosis in Santo Domingo, Dominican Republic: prevalence, clinical findings and response to antituberculosis treatment. J Acquir Immune Defic Syndr Hum Retroviral 1996; 13: 155-9.

8.- Barbagallo J, Tager P, Ingleton R, Hirsch R, Weinberg J. Cutaneous tuberculosis: Diagnosis and treatment. Amer $\mathrm{J}$ Clin Dermatol 2002; 3: 319-28.

9.- Jellis J E. Human immunodeficiency virus and osteoarticular tuberculosis. Clin Orthoped \& Relat Res 2002; 1: 27-31.

10.- Chan S, Birnbaum J, Rao M, Steiner P. Clinical manifestation and outcome of tuberculosis in children with acquired immunodeficiency syndrome. Pediatr Infect Dis J 1996; 15: 443-7

11.- Garay J. Clinical presentation of pulmonary tuberculosis in under $10 \mathrm{~s}$ and differences in AIDS-related cases: a cohort study of 115 patients. Trop Doct 1997; 27: 139-42.

12.- Mofenson L, Oleske J, Serchuck L, Van Dyke R, Wilfert C. Treating opportunistic infections among HIV-exposed adults. MMWR Morbid Mortal Wkly Rep 2004; 53(RR 14); 1-63.

13.- Thomson R. Laboratory diagnosis of respiratory infections. Curr Opin Infect Dis 1999; 12: 115-9.

14.- Wang S, Tay L. Early identification of Mycobacterium tuberculosis complex in BACTEC cultures by ligase chain reaction. J Med Microbiol 2002; 51: 710-2.

15.- Venkataraman P, Herbert D, Paramasivan C. Evaluation of the Bactec radiometric method in the early diagnosis of tuberculosis. Indian J Med Res 1998; 108: 120-7.

16.- Kampmann B, Douglas Y. Childhood tuberculosis: advances in immunopathogenesis, treatment and prevention. Curr Opin Infect Dis 1998; 11: 331-5.

17.- Havlir D, Barnes P Current concepts: Tuberculosis in patients with human immunodeficiency virus infection. N Engl J Med 1999; 340: 367-73.

18.- Ferrer J. Tuberculous pleural effusion and tuberculous empyema. Sem Resp Crit Care Med 2001; 22: 637-46.
19. - Chan S, Birnbaum J, Rao M, Stenner P Clinical manifestation and outcome of tuberculosis in children with acquired immunodeficiency syndrome. Pediatr Infect Dis J 1996; 15: 443-7.

20.- Squire S, Davies P. Treatment for mycobacterial infections. Curr Opin Infect Dis 1998; 11: 159-62.

21.- Van Scoy R, Wilkowske C. Antimycobacterial therapy. Mayo Clin Proc 1999; 74: 1038-48.

22.- Kindelán J. Síndrome de reconstitución inmunológica. Enf Emerg 2003; 5: 214-6.

23.- Furrer H, Malinverni R. Systemic inflammatory reaction after starting highly active antiretroviral therapy in AIDS patients treated for extrapulmonary tuberculosis. Amer J Med 1999; 106: 371-2.

24.- Hirch H, Kaufmanm G, Sendi P, Battegay M. Immune reconstitution in HIV infected patients. Clin Infect Dis 2004; 38: 1159-66.

25.- Snider D, Castro K. The global threat of drug-resistant tuberculosis. N Engl J Med 1998; 338: 1689-90.

26.- MMWR Morbid Mortal Wkly Rep, 2006; 55 (11): 301-5. Emergence of Mycobacterium tuberculosis with extensive resistance to second-line drugs- worldwide 2000-2004. 